\title{
Symmetric Controller Design for Symmetric Plant Using Matrix Inequality Conditions
}

\author{
Tomoyuki Nagashio and Takashi Kida
}

\begin{abstract}
For symmetric systems, the useage of symmetric controllers is known to be effective. The existence conditions of the controller have been shown in several studies. They require the symmetry of the plant as well as the control specifications. This paper describes the condition by matrix inequalities and relaxes it to be applicable to the case of non-symmetric sepcifications for the symmetric $\mathbf{H}_{\infty}$ controller design. Additionally, the optimal symmetric controller design for mechanical systems is investigated as a special case.
\end{abstract}

\section{INTRODUCTION}

Symmetric controller is known to be effective for the symmetric plants [1]. This is because it is regarded as superior to the non-symmetric controller in the robustness and the control performance when the plant is symmetric. For the controller synthesis, several schemes have been proposed [2], [3], [4], [5], [6], [7], including our study on the optimal $\mathrm{H}_{\infty}$ controller design for large space structures [8]. The literatures commonly use the fact that the symmetric controller exists for the plant having the symmetry with respect to the control input and measurement output as well as the exogenous input and controlled output. The controller is optimized for the generalized plant satisfying the symmetry in both senses using Riccati equations [3]. However, there exist some cases that non-symmetric control specifications should be used even when the plant is symmetric, for example the usage of the frequency weighting functions [9], which are generally non-symmetric, is effective in $\mathrm{H}_{\infty}$ controller design. In view of this, our first objective of this paper is to clarify the existence condition of the symmetric controller and describe it by matrix inequality conditions for the case of non-symmetric control specifications. The condition for the case of symmetric specification is derived from the result. Then, the discussion is focused to a specific class of systems for which non-parametric stability is guaranteed by the symmetric controller [2], for example the collocated mechanical system and an optimal $\mathrm{H}_{\infty}$ controller design method is derived.

\section{PROPERTY OF SYMMETRIC CONTROL SYSTEM}

First, we describe the properties of symmetric control systems. Let a controllable and observable system described by the state equation be given as

This work was not supported by any organization

T. Nagashio is with Department of Mechanical Engineering and Intelligent Systems, University of Electro Communications, Chofu, Tokyo, Japan nagasio@mce.uec.ac.jp

T. Kida is with Department of Mechanical Engineering and Intelligent Systems, University of Electro Communications, Chofu, Tokyo, Japan kida@mce.uec.ac.jp

$$
\mathcal{G}:\left\{\begin{aligned}
\dot{x} & =\mathcal{A} x+\mathcal{B} u \\
y & =\mathcal{C} x+\mathcal{D} u
\end{aligned}\right.
$$

where $x \in R^{p}$ is the state variable, $u \in R^{m}$ is the control input, and $y \in R^{m}$ is the measurement output. Laplace transform of $\mathcal{G}$ yields the transfer function matrix as

$$
G(s)=\mathcal{D}+\mathcal{C}(s I-\mathcal{A})^{-1} \mathcal{B}
$$

Additionally, let $\Sigma$ be a signature matrix which is diagonal with diagonal elements of +1 or -1 . Then, the following lemmas are known.

Lemma 1 [1] : The following conditions are equivalent.

1) $\mathcal{G}$ is symmetric.

2) $\Sigma G(s)=G^{T}(s) \Sigma, \forall s$

3) There exists a non-singular symmetric matrix $T$ such that

$$
T \mathcal{A}=\mathcal{A}^{T} T, \quad T \mathcal{B}=\mathcal{C}^{T} \Sigma, \quad \mathcal{D}=\Sigma \mathcal{D}^{T} \Sigma .
$$

Lemma 2 [1] : The system $\mathcal{G}$ is called internally symmetric with internal signature $T$ and external signature $\Sigma$ if $T$ belongs to the admissible class of signature matrix.

The symmetric property of the control system is generally defined by the input-output relationship of the system. Lemma 1 relates the symmetric structure to the state space realization. The conditions are effectively used in the following symmetric controller design.

\section{DESIGN OF SYMMETRIC CONTROLLER}

For the symmetric controller, several design schemes have been proposed [3]. They rely on the recognition that the symmetric controllers always exist when a non-symmetric controller exists for the plant, if the control specifications of the closed-loop system as well as the plant are symmetric. In the $\mathrm{H}_{\infty}$ control problem, this statement requires that the exogenous input and the controlled output must be selected to satisfy the symmetry. However, the condition constrains the design flexibility. In view of this, we consider the condition that the symmetric $\mathrm{H}_{\infty}$ controller exists for the symmetric plant with the non-symmetric control specifications.

For the design of the controller, the following generalized plant including (1) is considered by setting $A=\mathcal{A}, B_{2}=\mathcal{B}$, $C_{2}=\mathcal{C}$ and $\mathcal{D}=0$ in (1).

$$
\begin{aligned}
\dot{x} & =A x+B_{1} w+B_{2} u \\
z & =C_{1} x+D_{11} w+D_{12} u \\
y & =C_{2} x+D_{21} w
\end{aligned}
$$


It is assumed that $A, B_{2}$ and $C_{2}$ satisfy the condition (3) as

$$
T A=A^{T} T, \quad T B_{2}=C_{2}^{T} .
$$

This is the condition of the plant itself being symmetric. Other matrices $B_{1}, C_{1}, D_{11}, D_{12}$ and $D_{21}$ corresponding to the exogenous input $w$ and the controlled output $z$ are appropriately defined. When these matrices also satisfy the condition (3) as

$$
T B_{1}=C_{1}^{T}, \quad D_{11}=D_{11}^{T} \quad D_{12}=D_{21}^{T},
$$

the control specifications are said to be symmetric. For the generalized plant (4), we use the dynamic output feedback controller

$$
\begin{aligned}
\dot{x}_{k} & =A_{k} x_{k}+B_{k} y \\
u & =C_{k} x_{k}+D_{k} y
\end{aligned}
$$

where $x_{k} \in R^{k}$ is the state variable of the controller, and $B_{k}$ and $C_{k}$ are assumed to have full ranks. By applying (7) to (4), the closed-loop system becomes

$$
\begin{aligned}
\dot{x}_{c l} & =A_{c l} x_{c l}+B_{c l} w \\
z & =C_{c l} x_{c l}+D_{c l} w
\end{aligned}
$$

where $x_{c l}=\left[\begin{array}{ll}x^{T} & x_{k}^{T}\end{array}\right]^{T}$, and

$$
\begin{aligned}
A_{c l} & =\left[\begin{array}{cc}
A+B_{2} D_{k} C_{2} & B_{2} C_{k} \\
B_{k} C_{2} & A_{k}
\end{array}\right] \\
B_{c l} & =\left[\begin{array}{c}
B_{1}+B_{2} D_{k} D_{21} \\
B_{k} D_{21}
\end{array}\right] \\
C_{c l} & =\left[\begin{array}{cc}
C_{1}+D_{12} D_{k} C_{2} & D_{12} C_{k}
\end{array}\right] \\
D_{c l} & =\left[D_{11}+D_{12} D_{k} D_{21}\right]
\end{aligned}
$$

Then, the bounded real lemma in the $\mathrm{H}_{\infty}$ control theory is given as follows.

Lemma 3 [10] : The following statements are equivalent.

1) The matrix $A_{c l}$ is internally stable and the $\mathrm{H}_{\infty}$ norm of the closed-loop system (8) is less than a scalar $\gamma>0$.

2) There exists a matrix $X_{c l}=X_{c l}^{T}>0$ satisfying

$$
\mathcal{H}=\left[\begin{array}{ll}
\mathcal{H}_{11} & \mathcal{H}_{12} \\
\mathcal{H}_{12}^{T} & \mathcal{H}_{22}
\end{array}\right]<0
$$

where

$$
\begin{aligned}
\mathcal{H}_{11} & =X_{c l} A_{c l}+A_{c l}^{T} X_{c l} \\
\mathcal{H}_{12} & =\left[\begin{array}{ll}
X_{c l} B_{c l} & C_{c l}^{T}
\end{array}\right] \\
\mathcal{H}_{22} & =\left[\begin{array}{cc}
-\gamma I & D_{c l}^{T} \\
D_{c l} & -\gamma I
\end{array}\right] .
\end{aligned}
$$

Our objective in this section is to obtain the controller (7) satisfying (10) and

$$
T_{k} A_{k}=A_{k}^{T} T_{k}, \quad T_{k} B_{k}=C_{k}^{T}, \quad D_{k}=D_{k}^{T}
$$

under the conditions (5) or (6). In (12), $T_{k}$ corresponds to the non-singular symmetric matrix in Lemma 1 . We consider to use the following lemmas to the problem.
Lemma 4 : For any generalized plant (4), there exists a positive definite symmetric matrix $X_{c l}$ and a scalar $\gamma$ satisfying (10), if $A_{c l}$ is internally stable.

Proof: When $A_{c l}$ is internally stable, $\mathcal{H}_{11}<0$ holds from the Lyapunov inequality. Then, for sufficiently large $\gamma$, there exists $\mathcal{H}_{22}<0$ that satisfies the Schur complement

$$
-\mathcal{H}_{11}+\mathcal{H}_{12} \mathcal{H}_{22}^{-1} \mathcal{H}_{12}^{T}>0
$$

Therefore, there exists $X_{c l}$ satisfying (10).

Lemma 5 : There exists a symmetric controller that guarantees the internal stability of the closed-loop system (8) under the conditions (5) and (12), if the inequality $\mathcal{H}_{11}<0$ is feasible and its solution $X_{c l}$ satisfies the following.

$$
X_{c l}^{-1}=Y_{c l}=T_{c l}^{-1} X_{c l} T_{c l}^{-1}
$$

where $T_{c l}=\operatorname{diag}\left[T T_{k}\right]$.

Proof : By multiplying $T_{c l}^{-1}$ to both sides of $\mathcal{H}_{11}$, we obtain

$$
\begin{aligned}
& T_{c l}^{-1} X_{c l} T_{c l}^{-1} T_{c l} A_{c l} T_{c l}^{-1}+* \\
& =T_{c l}^{-1} X_{c l} T_{c l}^{-1}\left[\begin{array}{cc}
A^{T}+C_{2}^{T} D_{k}^{T} B_{2}^{T} & C_{2}^{T} B_{k}^{T} \\
C_{k}^{T} B_{2}^{T} & A_{k}^{T}
\end{array}\right]+* \\
& =Y_{c l} A_{c l}^{T}+A_{c l} Y_{c l}<0
\end{aligned}
$$

where $*$ denotes the transpose of the first term. Therefore, the symmetric controller based on Lemma 1 guarantees the internal stability, if $\mathcal{H}_{11}<0$ is feasible and the condition (14) is satisfied.

From the lemmas, we can state the following theorem.

Theorem 1 : There exists a symmetric $\mathrm{H}_{\infty}$ controller satisfying (10) under the conditions (5) and (12), if the closed-loop system (8) is internally stable and the condition (14) is satisfied.

Proof: It is obvious from Lemmas 4 and 5.

Theorem 1 states that there exists a bounded scalar $\gamma>0$ satisfying (10) and we can obtain the symmetric $\mathrm{H}_{\infty}$ controller even when the control specification is not symmetric if the closed-loop system is internally stable. However, from the practical viewpoint, the $\mathrm{H}_{\infty}$ performance may be degraded because of the increase of $\gamma$.

For the optimal symmetric $\mathrm{H}_{\infty}$ controller design, we can derive the following corollary from Theorem 1 .

Corollary 1 : There exists the symmetric $\mathrm{H}_{\infty}$ controller satisfying (10) under the conditions (5) and (12), if and only if the matrices $X$ and $Y$ satisfy

$$
\begin{aligned}
& N_{R}^{\perp}\left[\begin{array}{ccc}
X A+A^{T} X & X B_{1} & C_{1}^{T} \\
B_{1}^{T} X & -\gamma I & D_{11}^{T} \\
C_{1} & D_{11} & -\gamma I
\end{array}\right] N_{R}^{\perp T}<0 \\
& N_{R}^{\perp}\left[\begin{array}{ccc}
A Y+Y A^{T} & Y C_{1}^{T} & B_{1} \\
C_{1} Y & -\gamma I & D_{11} \\
B_{1}^{T} & D_{11}^{T} & -\gamma I
\end{array}\right] N_{R}^{\perp T}<0 \\
& {\left[\begin{array}{cc}
X & I \\
I & Y
\end{array}\right] \geq 0, \quad \operatorname{rank}\left[\begin{array}{cc}
X & I \\
I & Y
\end{array}\right] \leq n+p}
\end{aligned}
$$


where

$$
\begin{aligned}
X_{c l} & =\left[\begin{array}{cc}
X & X_{2} \\
X_{2}^{T} & X_{3}
\end{array}\right] \\
Y_{c l} & =\left[\begin{array}{cc}
Y & Y_{2} \\
Y_{2}^{T} & Y_{3}
\end{array}\right]=\left[\begin{array}{cc}
T^{-1} X T^{-1} & T^{-1} X_{2} T_{k}^{-1} \\
T_{k}^{-1} X_{2}^{T} T^{-1} & T_{k}^{-1} X_{3} T_{k}^{-1}
\end{array}\right]
\end{aligned}
$$

and the matrices $N_{R}^{\perp}$ and $N_{L}^{\perp}$ are the bases of the null spaces of $N_{R}=\left[\begin{array}{lll}C_{2} & D_{21} & 0\end{array}\right]^{T}$ and $N_{L}=\left[\begin{array}{lll}B_{2}^{T} & D_{12}^{T} & 0\end{array}\right]^{T}$, respectively.

Proof: It is derived by applying Lemma 5 to the standard algorithm for $\mathrm{H}_{\infty}$ controller design using the LMIs [11].

From Corollary 1, the symmetric controller can be designed by the convex optimization in the general $\mathrm{H}_{\infty}$ controller design method. In the general case, we might not be able to obtain the solutions of (16), because the closedloop system is not always internally stable under only the conditions (5), (12) and (14). However, for the limited class of the symmetric system, the internal stability can be guaranteed by the property of the symmetric structure as described in the next section.

On the other hand, if the generalized plant (4) also satisfies the symmetric control specifications (6), the following well known fact [3] is derived from Theorem 1.

Corollary 2 : There also exists a symmetric $\mathrm{H}_{\infty}$ controller, if there exists a non-symmetric $\mathrm{H}_{\infty}$ controller for (4) under the conditions (5) and (6).

In order to clarify the relationship of matrix inequality and Corollary2, its proof is given below.

Proof : Assume that there exists a non-symmetric $\mathrm{H}_{\infty}$ controller under the conditions (5) and (6). The matrix inequality (10) can be described as

$$
\mathcal{H}=Q_{x}+C_{e}^{T} S^{T} B_{e}^{T} X_{e}+X_{e} B_{e} S C_{e}<0
$$

where

$$
\begin{aligned}
& Q_{x}=\left[\begin{array}{ccc}
X_{c l} A_{s}+A_{s}^{T} X_{c l} & X_{c l} B_{s} & C_{s}^{T} \\
B_{s}^{T} X_{c l} & -\gamma I & D_{s}^{T} \\
C_{s} & D_{s} & -\gamma I
\end{array}\right] \\
& X_{e}=\operatorname{diag}\left[\begin{array}{lll}
X_{c l} & I & I
\end{array}\right], \quad S=\left[\begin{array}{ll}
A_{k} & B_{k} \\
C_{k} & D_{k}
\end{array}\right] \\
& B_{e}^{T}=\left[\begin{array}{lll}
B_{e 1}^{T} & 0 & B_{e 2}^{T}
\end{array}\right], \quad C_{e}=\left[\begin{array}{lll}
C_{e 1} & 0 & C_{e 2}
\end{array}\right] \\
& A_{s}=\left[\begin{array}{cc}
A & 0 \\
0 & 0
\end{array}\right], \quad B_{s}=\left[\begin{array}{c}
B_{1} \\
0
\end{array}\right] \\
& C_{s}=\left[\begin{array}{ll}
C_{1} & 0
\end{array}\right], \quad D_{s}=D_{11} \\
& B_{e 1}=\left[\begin{array}{cc}
0 & B_{2} \\
I & 0
\end{array}\right], \quad B_{e 2}=\left[\begin{array}{ll}
0 & D_{12}
\end{array}\right] \\
& C_{e 1}=\left[\begin{array}{cc}
0 & I \\
C_{2} & 0
\end{array}\right], \quad C_{e 2}=\left[\begin{array}{c}
0 \\
D_{21}
\end{array}\right]
\end{aligned}
$$

Then, by the congruent transformation, the following matrix inequality is obtained.

$$
X_{t} \mathcal{H} X_{t}=Q_{y}+B_{e} S^{T} C_{e} Y_{e}+Y_{e} C_{e}^{T} S B_{e}^{T}<0
$$

where

$$
\begin{aligned}
& X_{t}=\operatorname{diag}\left[T_{t} X_{c l} T_{t} I I\right], \quad T_{t}=\left[\begin{array}{cc}
T^{-1} & 0 \\
0 & I
\end{array}\right] \\
& Y_{e}=\operatorname{diag}\left[\begin{array}{lll}
Y_{s} & I
\end{array}\right], \quad Y_{s}=T_{t} X_{c l} T_{t}=X_{c l}^{-1} \\
& Q_{y}=\left[\begin{array}{ccc}
Y_{s} A_{s}^{T}+A_{s} Y_{s} & Y_{s} C_{s}^{T} & B_{s} \\
C_{s} Y_{s} & -\gamma I & D_{s}^{T} \\
B_{s}^{T} & D_{s} & -\gamma I
\end{array}\right]
\end{aligned}
$$

By multiplying $Y_{e}^{-1}$ to both sides of (20), the matrix inequality

$$
Q_{x}+X_{e} B_{e} S^{T} C_{e}+C_{e}^{T} S B_{e}^{T} X_{e}<0
$$

is derived. From (18) and (22), we obtain the following feasible condition.

$$
2 Q_{x}+X_{e} B_{e}\left(S+S^{T}\right) C_{e}+C_{e}^{T}\left(S+S^{T}\right) B_{e}^{T} X_{e}<0
$$

Therefor, there exists a symmetric $\mathrm{H}_{\infty}$ controller under the conditions (5) and (6), if there exists a non-symmetric $\mathrm{H}_{\infty}$ controller.

\section{SYMMETRIC MECHANICAL SYSTEM}

In this section, we apply Theorem 1 to the mechanical system which belongs to the specific class of the symmetric system. We consider the mechanical systems, described by the dynamics equation

$$
M \ddot{q}+D \dot{q}+K q=L u+F w
$$

where $q \in R^{n}$ is the displacement vector, $u \in R^{m}$ is the control input vector, and $w \in R^{l}$ is the disturbance input vector. Matrices $M, D$ and $K \in R^{n \times n}$ denote mass, damping and stiffness, respectively, and generally satisfy

$$
M=M^{T}>0, \quad D=D^{T} \geq 0, \quad K=K^{T} \geq 0 .
$$

Positive semi-definiteness implies that the system has rigid body modes corresponding to the zero eigenvalues of $D$ and $K$. The matrices $L \in R^{n \times m}$ and $F \in R^{n \times l}$ are defined by the locations of actuators and the directions of actuator control forces and the points of application and directions of disturbance forces. It is assumed that sensors and actuators are collocated and displacements are measured at the actuator locations. In this case, the measurement output $y \in R^{m}$ is described as

$$
y=L^{T} p+N w .
$$

In addition, we assume that

$$
\operatorname{rank}\left[\begin{array}{ll}
D & L
\end{array}\right]=n, \quad \operatorname{rank}\left[\begin{array}{ll}
K & L
\end{array}\right]=n .
$$

This is the requirement for the rigid body modes of (24) being controllable and observable [3]. The type of the system includes the linearized large flexible spacecrft or multi-body mechanical system, for which many robust control method have been investigated. 
First, we show that the system satisfies Lemma 1. For the purpose, (24) and (26) are described by the following state equation when $w=0$.

$$
\begin{aligned}
\dot{x} & =A x+B_{2} u \\
y & =C_{2} x
\end{aligned}
$$

where $x=\left[\begin{array}{ll}q^{T} & \dot{q}^{T}\end{array}\right]^{T}$ is the state variable and matrices $A, B_{2}$ and $C_{2}$ are as follows.

$$
\begin{aligned}
& A=\left[\begin{array}{cc}
0 & I \\
-M^{-1} K & -M^{-1} D
\end{array}\right], \quad B_{2}=\left[\begin{array}{c}
0 \\
M^{-1} L
\end{array}\right] \\
& C_{2}=\left[\begin{array}{ll}
L^{T} & 0
\end{array}\right]
\end{aligned}
$$

This state equation satisfies Lemma 1 when we select

$$
\begin{aligned}
& T A=A^{T} T, \quad T B_{2}=C_{2}^{T} \Sigma \\
& \Sigma=I, \quad T=\left[\begin{array}{cc}
D & M \\
M & 0
\end{array}\right] .
\end{aligned}
$$

By applying the symmetric controller (7) to (28), the closedloop system becomes

$$
\dot{x}_{c l}=A_{c l} x_{c l}
$$

where $x_{c l}=\left[\begin{array}{ll}x^{T} & x_{k}^{T}\end{array}\right]^{T}$ and

$$
\begin{aligned}
& A_{c l}=\left[\begin{array}{cc}
A+B_{2} D_{k} C_{2} & B_{2} C_{k} \\
B_{k} C_{2} & A_{k}
\end{array}\right] \\
& 0 \\
& =\left[\begin{array}{ccc}
0 & I & 0 \\
-M^{-1}\left(K+L D_{k} L^{T}\right) & -M^{-1} D & M^{-1} L C_{k} \\
B_{k} L^{T} & 0 & A_{k}
\end{array}\right]
\end{aligned}
$$

It satisfies the following salient stability property.

Theorem 2: The system (31) is stable if the following condition is satisfied.

$$
T_{k}>0, \quad\left[\begin{array}{cc}
T_{k} A_{k} & T_{k} B_{k} \\
C_{k} & D_{k}
\end{array}\right]=\mathcal{S}=\mathcal{S}^{T}<0
$$

Proof : Let a matrix

$$
V=\left[\begin{array}{ccc}
-D & -M & 0 \\
M & 0 & 0 \\
0 & 0 & -T_{k}
\end{array}\right],
$$

the following relationship is derived under the conditions (25), (27) and (33).

$$
\begin{aligned}
V A_{c l} & =\left[\begin{array}{ccc}
K-L D_{k} L^{T} & 0 & -L C_{k} \\
0 & M & 0 \\
-T_{k} B_{k} L^{T} & 0 & -T_{k} A_{k}
\end{array}\right] \\
& =\left[\begin{array}{ccc}
K-L D_{k} L^{T} & 0 & -L C_{k} \\
0 & M & 0 \\
-C_{k}^{T} L^{T} & 0 & -T_{k} A_{k}
\end{array}\right]=A_{c l}^{T} V^{T}>0
\end{aligned}
$$

Therefore, by defining the positive definite symmetric matrix

$$
X_{c l}=V^{T}\left(V A_{c l}\right)^{-1} V=X_{c l}^{T}>0,
$$

we obtain the following Lyapunov inequality.

$$
\begin{aligned}
& X_{c l} A_{c l}+A_{c l}^{T} X_{c l} \\
& \quad=V^{T}+V=-W=-\operatorname{diag}\left[\begin{array}{lll}
2 D & 0 & 2 T_{k}
\end{array}\right] \leq 0 .
\end{aligned}
$$

Then, from the conditions (25), (27) and

$$
\begin{aligned}
\operatorname{rank}\left[\begin{array}{c}
A_{c l} \\
W
\end{array}\right]= & \operatorname{rank}\left(K+L D_{k} L^{T}\right) \\
& +\operatorname{rank} M+\operatorname{rank} A_{k}=\operatorname{dim} x_{c l},
\end{aligned}
$$

the pair $\left(W, A_{c l}\right)$ is observable. Therefore, the system (31) is stable under the condition (33).

Theorem 2 means that the symmetric controller satisfying (33) guarantees the closed-loop stability based only on the symmetric structure of the mechanical system (31) and the controller (7). This is effective to satisfy the internal stability condition in Theorem 1. Therefore, by applying Theorem 1 to the generalized plant (4) including (24) and (26), we can obtain the optimal symmetric $\mathrm{H}_{\infty}$ controller satisfying suitable control specification under the condition (33).

\section{CONCLUSION}

We have derived some conditions on the symmetric $\mathrm{H}_{\infty}$ controller design in the form of matrix inequalities. Additionally, its applications to collocated mechanical systems have been investigated. The motivation of our study is to improve the design flexibility of the symmetric controller, especially by relaxing the symmetry constraint of the control specification. For the design purposes, the obtained conditions have been described by LMIs.

To extend our scheme for the frequency shaping method in the $\mathrm{H}_{\infty}$ control theory is currently under study. The result is going to be applied to the attitude control orbit experiment of a large flexible spacecraft.

\section{REFERENCES}

[1] J. C. Willems, "Realization of System with Internal Passivity and Symmetry Constraints", J. Franklin Institute, vol. 301, no. 6, pp 605621, 1976.

[2] Y. Fujisaki, M. Ikeda and K. Miki, "Robust Stabilization of Large Space Structures Via Displacement Feedback", IEEE Trans. Automat. Contr., vol. 46, pp 1993-1996, 2001.

[3] M. Ikeda and K. Takeuchi, "Controller Design for Space Structures with Collocated Sensors and Actutors," Trans. of SICE (in Japanese), vol. 30, no. 10, pp 1209-1215, 1994.

[4] L. Fortuna, G. Muscato and G. Nunnari, "On $\mathrm{H}_{\infty}$ Control for Symmetric System", Proc. Conf. Dec. Contr, Tucson, Arizona, December, pp 3723-3725, 1992.

[5] A. Arbel and N. K. Gupta, "Robust Colocated Control for Large Flexible Space Structures," AIAA J. Guidance and Control, vol. 4, no. 5, pp 480-486, 1981.

[6] E. E. Zajac, "The Kelvin-Tait-Chetaev Theorem and Extensins," $J$. Astronautical Sci., vol. 11, no. 2, pp 46-49, 1964.

[7] S. M. Joshi, "Robust Properties of Collocated Controllers for Flexible Spacecraft," AIAA J. Guidance and Control, vol. 9, no. 1, pp 85-91, 1986.

[8] T. Nagashio and T. Kida, "Robust Control of Flexible Mechanical Systems by Utilizing Symmetry and Its Application to Large Space Structures", Proc. of IEEE International CCA, Taipei, Taiwan, September 2-4, pp 418-424, 2004.

[9] N. Zhou, J. C. Doyle and K. Glover, "Robust and Optimal Control", Prentice Hall, 1996.

[10] S. Boyd, L. E. Ghaoui, E. Feron and V. Balakrishnan, "Linear Matrix Inequalities in System and Control Theory," SIAM Studies in Applied Mathematics, vol. 15, 1994.

[11] T. Iwasaki and R. E. Skelton, "All Controllers for the General $\mathrm{H}_{\infty}$ Cotrol Problem : LMI Existence Conditions and State Space Formulas," Automatica, vol. 30, no. 8, pp 1307-1317, 1994. 